\author{
Paolo Navalesi \\ Massimo Antonelli \\ Giorgio Conti
}

\section{From belief to knowledge: call it evidence if you prefer}

Received: 3 October 2010

Accepted: 4 October 2010

Published online: 10 November 2010

(C) Copyright jointly held by Springer and ESICM 2010

This editorial refers to the article available at: doi:10.1007/s00134-010-2066-3.

P. Navalesi (๘)

Anesthesia and Intensive Care,

Dipartimento di Medicina Clinica e Sperimentale,

Università del Piemonte Orientale "A. Avogadro",

Alessandria-Novara-Vercelli, Italy

e-mail: paolo.navalesi@med.unipmn.it

Tel.: +39-0321-3733406

Fax: +39-0321-3733406

M. Antonelli · G. Conti

Department of Intensive Care and Anesthesia,

Catholic University of Rome, Rome, Italy

Noninvasive ventilation (NIV) is nowadays one of the therapeutic possibilities for ICU physicians because it has been shown to be effective in achieving the aims of mechanical ventilation, while avoiding the risks associated with endotracheal intubation [1].

The major difference between invasive and noninvasive ventilation relies on the type of interface connecting the patient to the ventilator. The noninvasive interface is the major determinant of the advantages of this technique, such as improving patient's tolerance of mechanical ventilation, eliminating the need for heavy sedation, and decreasing the risk for infections in general and for ventilator-associated pneumonia in particular. The interface, however, is also the major determinant of NIV disadvantages, complications, and side effects. The choice of the interface is therefore of paramount importance for the management of NIV, dramatically affecting the rate of success and reducing complications and side effects related to the technique [1].

NIV interfaces can be classified as the oral interface (mouthpiece), nasal interface (internal and external), oronasal masks (including nose and mouth), full face masks (incorporating the eyes), and helmet (incorporating the whole head). While oral and nasal interfaces are preferentially used for chronic patients receiving long-term domiciliary NIV, the other interfaces are preferred for dyspneic patients with ARF who breath through both the nose and mouth.

NIV has in general a good outcome, but failure may exceed $50 \%$ in the most severe cases of ARF [2, 3]. Discomfort and lack of improvement in arterial blood gases are the most common causes for NIV failure $[2,3]$. Patient discomfort may be generated by various problems such as air leaks, skin breakdown, claustrophobia, or eye irritation [1]. Nowadays, the large availability of different shapes, construction materials, and fixation systems should make it easy to find the most appropriate interface for most of the patients. Economic and logistic reasons, however, may restrict the availability of interfaces for the clinician and favor the choice for full face masks and helmets, as they fit the majority of individuals.

The internal space of the interfaces has long constituted a major concern as it is considered an additional dead space capable of causing substantial re-breathing, interfering with effective ventilation, especially in hypercapnic patients [4]. This notion has been repeatedly challenged by physiological and clinical studies. In a bench investigation, Saatci et al. [5] found that the increase in the actual dead space observed during spontaneous unassisted breathing consequent to the application of oro-nasal masks was reduced close to zero thanks to the continuous flow produced by turbine ventilators throughout expiration. 
In this issue of Intensive Care Medicine, Fodil et al. [6] present a new evaluation of the actual dead space of four interfaces in common clinical use (two oro-nasal masks, one full face mask, and a helmet) that have different internal volumes, ranging from about $100 \mathrm{ml}$ to more than $10 \mathrm{l}$. The authors used mathematical simulations made with a computational fluid dynamics software analyzing the pressure field/flow pattern and variations in gas composition inside the interface. It was found that, in spite of the large variation of their internal volume and consequent to the streaming effect of gas flowing through the interface, the actual dead space of the four interfaces differed to a relatively moderate extent, ranging from 110 to $370 \mathrm{ml}$. The effective dead space was very close to their internal volume for the two oro-nasal masks, while it approached half the tidal volume, irrespective of the internal volume, for the full face mask and the helmet.

While confirming the results previously obtained by Saatchi et al. [5] using a different technique, the study by Fodil et al. [6] extends the analysis to the helmet, an interface that, despite increasing popularity because of its excellent tolerability over time [7], still raises concern because of the high risk of re-breathing related to its large internal volume. Fodil et al. [6] elegantly show that when the internal volume is much larger than the tidal volume, as occurs with the helmet, the importance of the convective flows is negligible and is only minimally affected by the breathing cycle. The helmet's actual dead space is thus about $50 \%$ of the tidal volume in all instances. Conversely, the convective flows appeared to be important for the oro-nasal masks having an internal volume relatively smaller than the tidal volume. With this interface the internal gas volume is washed out by the inhaled and exhaled flow, and the dead space approximates the internal volume. The actual dead space of the full face mask depends on both convective and diffusive phenomena and can be remarkably affected by differences in the geometric shape of the interface. This characteristic makes generalizing the results obtained by Fodil et al. to all full face masks impossible. It is noteworthy that in the setup of this study, a continuous gas flow of $300 \mathrm{ml} / \mathrm{s}$ was delivered throughout the expiratory phase of the breathing cycle; the presence and the extent of this flow when applying positive end-expiratory pressure (PEEP) may substantially vary among ventilators, depending on their operation functioning and on the settings adopted. This might in turn also affect the actual dead space of the interface.

The findings of this study allow us to better understand and reconcile the results of previous physiologic and clinical studies [7, 8]. In particular, these data suggest that the effective dead space is influenced by the breathing pattern: increasing the tidal volume decreases the influence of the internal volume of the small interfaces, whereas lowering it makes the larger interfaces paradoxically more efficient. This is of particular clinical relevance in the pediatric field where internal dead space and re-breathing have been always considered of major importance, with few masks available for children, and even fewer for infants. Because NIV is now routinely utilized in the pediatric field [9], the development of interfaces specifically designed for infants is urgent, and the results from Fodil et al. are extremely important for NIV application to pediatric patients.

The technique presented by Fodil et al. [6] might be important also for manufacturers, by allowing them to predict the behavior of their devices in different conditions. In this regards, we wonder whether it is time for the producers of NIV interfaces to provide standardized bench data obtained with a reproducible technique, like the one presented in this elegant study.

Deceptive assumptions, based on simplistic interpretation of facts, often lead us to consider a belief as the truth. The truth is the opposite of deception and requires knowledge of the mechanisms underlying the observed facts.

Call it evidence, if you prefer.

\section{References}

1. Mehta S, Hill NS (2010) Noninvasive ventilation. Am J Respir Crit Care Med 163:540-577

2. Squadrone E, Frigerio P, Fogliati C, Gregoretti C, Conti G, Antonelli M, Costa R, Baiardi P, Navalesi P (2004) Noninvasive vs invasive ventilation in COPD patients with severe acute respiratory failure deemed to require ventilatory assistance. Intensive Care Med 30:1303-1310
3. Antonelli M, Conti G, Moro ML, Esquinas A, Gonzalez-Diaz G, Confalonieri M, Pelaia P, Principi T, Gregoretti C, Beltrame F, Pennisi MA, Arcangeli A, Proietti R, Passariello M, Meduri GU (2001) Predictors of failure of noninvasive positive pressure ventilation in patients with acute hypoxemic respiratory failure: a multicenter study. Intensive Care Med 27:1718-1728
4. Ferguson GT, Gilmartin M (1995) CO2 rebreathing during BiPAP ventilatory assistance. Am J Respir Crit Care Med 151:1126-1135

5. Saatci E, Miller DM, Stell IM, Lee KC, Moxham J (2004) Dynamic dead space in face masks used with noninvasive ventilators: a lung model study. Eur Respir J 23:129-135

6. Fodil R, Lellouche F, Mancebo J, Isabey D, Brochard L, Louis B (2011) Comparison of patient-ventilator interfaces based on their computerized effective dead space. Intensive Care Med. doi:10.1007/s00134-010-2066-3 
7. Antonelli M, Pennisi MA, Pelosi P, Gregoretti C, Squadrone V, Rocco M, Cecchini L, Chiumello D, Severgnini P, Proietti R, Navalesi P, Conti G (2004) Noninvasive positive pressure ventilation using a helmet in patients with acute exacerbation of chronic obstructive pulmonary disease: a feasibility study. Anesthesiology 100:16-24
8. Fraticelli AT, Lellouche F, L'Her E, Taille S, Mancebo J, Brochard L (2009) Physiological effects of different interfaces during noninvasive ventilation for acute respiratory failure. Crit Care Med 37:939-945
9. Piastra M, De Luca D, Pietrini D, Pulitano S, D'Arrigo S, Mancino A, Conti G (2009) Noninvasive pressuresupport ventilation in immunocompromised children with ARDS: a feasibility study. Intensive Care Med 35:1420-1427 